\title{
Pulmonary thromboembolism associated with Klippel Trenaunay Weber Syndrome: A rare case report
}

\author{
Talib S H${ }^{1}$, Bhattu S R ${ }^{2}$, Punde Gaurav ${ }^{3}$, Hegde Rohan ${ }^{3}$, \\ Sarode Vinit ${ }^{3}$, Deshmukh Shridhar ${ }^{3}$ \\ ${ }^{l}$ Professor \& Head of Medicine, Department of Medicine MGM Medical College, Aurangabad, 431003, India \\ ${ }^{2}$ Associate professor, Department of Medicine MGM Medical College, Aurangabad, 431003, India \\ ${ }^{3}$ Post graduate Residents in Medicine, Department of Medicine MGM Medical College, \\ Aurangabad, 431003, India
}

\begin{abstract}
Klippel Trenaunay weber syndrome is a rare disease of vascular and lymphatic system presenting with port wine nevus, vascular malformations, hypertrophy of both bony and soft tissues. The patient may develop unexplained pulmonary hypertension among in such cases and may need screening for diagnosis of pulmonary embolism as observed in the present case.
\end{abstract}

Keywords - Klippel Trenaunay weber syndrome, port wine stain, pulmonary thromboembolism

\section{Introduction}

Klippel-Trénaunay syndrome (KTS), formerly Klippel Trénaunay Weber syndrome and sometimes angio osteohypertrophy syndrome or hemangiectatic hypertrophy ${ }^{1}$ is a rare congenital disorder characterised by triad of varicose veins, cutaneous hemangiomas and hypertrophy of soft tissue and bone. The other associated abnormality includes varying degree of lymphoedema. The disorder has risk of deep venous thrombosis and pulmonary embolism. In the world literature scanty case reports are available on the subjects, especially cases who have recognition of pulmonary embolism. We report our experience with young female patient having features of KWS and evidence of pulmonary embolism. Such subset of patients need early recognition and evaluation with aggressive approach.

\section{Case report}

A 26 year female hospitalized with complaints of shortness of breath, easy fatiguability \& edema of bilateral lower extremities. On examination blood pressure was $120 / 80 \mathrm{mmHg}$, pulse $110 / \mathrm{min}$ sinus rhythm, RR 24 cycles/min. On auscultation her lungs were clear. The Both lower extremities were edematous, equally affected with prominent superficial varicosities in medial and lateral aspects of legs were observed on standing. No bruise or thrill was found. Cutaneous Hemangioma of port wine type was observed extending from lateral aspect of right thigh to right lower back region along with right buttock (Fig1). Both feet had polydactyly and hypertrophy of bilateral lower limbs (Fig2). X ray lower limbs was suggestive of hypertrophy and asymmetric osteohypertrophy of the both feet. USG abdomen was normal. No Arteriovenous fistulas were noted in spleen and liver. 2D echo revealed acute cor pulmonale with moderate PAH with Dilated RA, RV, Main Pulmonary Artery \& Right Pulmonary Artery. The RVEF was $40 \%$ and PASP by TR jet was $49 \mathrm{mmhg}$ with elevated RV filling pressures and RV dysfunction, features consistent with pulmonary embolism. The patient then subjected to CT pulmonary angiogram which revealed partial lumen occluding pulmonary thrombosis in lobar and segmental branches of right middle and bilateral lower lobe along with small pulmonary infarct in postero basal segment of right lower lobe. Venous Doppler of bilateral lower limbs was suggestive of B/L saphenofemoral incompetency and poor flow reversal on Valsalva in standing and lying down position either side on venous Doppler showing venous impediment flow. She was started on warfarin .Her symptoms relived and patient discharged after 7 days with advice to continue warfarin with regular follow up. 


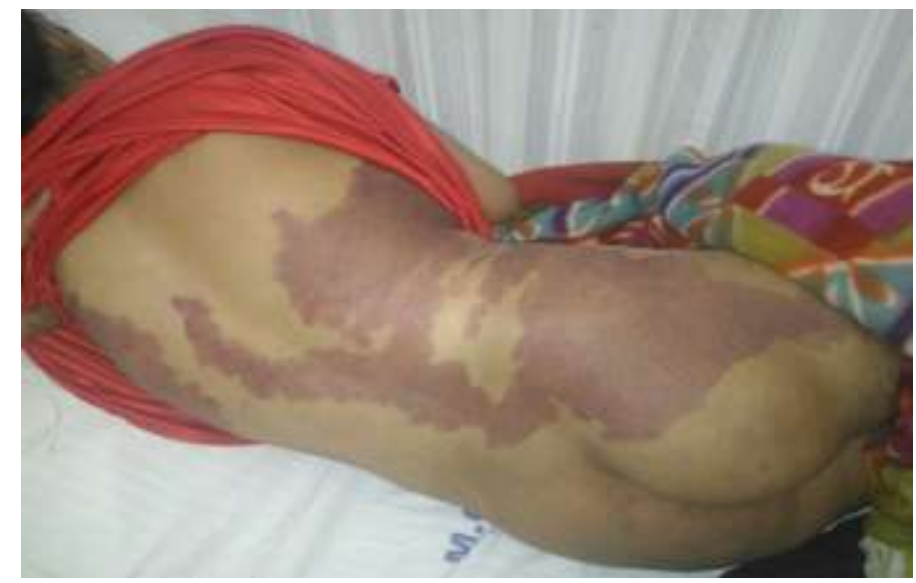

Figure1: showing port wine type hemangioma extending from lateral aspect of right thigh to right lower back region along with right buttock

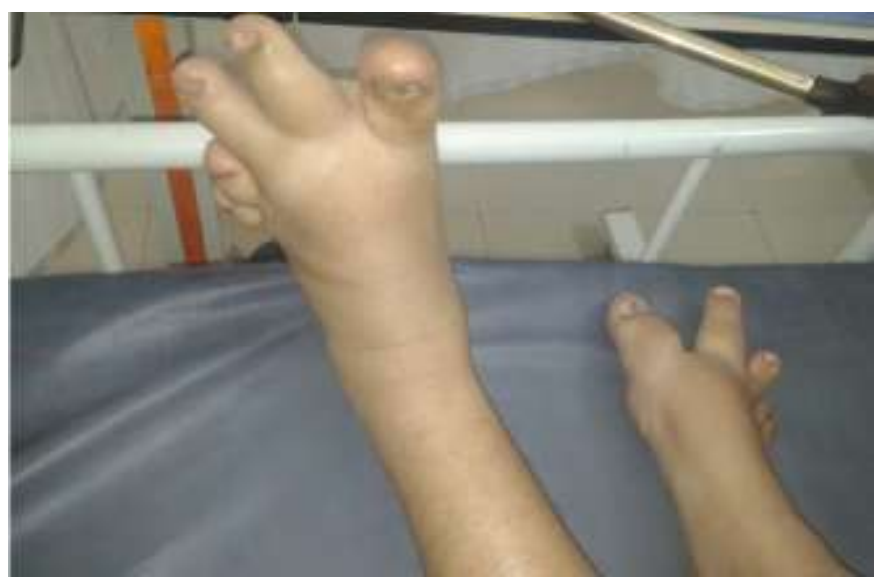

Figure 2: of $\mathrm{B} / \mathrm{L}$ feet with polydactyly and hypertrophy of bilateral lower limbs

\section{Discussion}

The classical Klippel Trenaunay weber syndrome (KWS) is a combination of cutaneous angiomatosis, varicose veins and bony and soft tissue hypertrophy described first by French physicians Klippel and Trenaunay in $1900^{2}$. Synonymously KTWS terminology used variably with different meanings. Unlike the Parkes weber syndrome, affected tissue do not contain hemodynamically significant arteriovenous communication, but other soft tissue, lymphatic and bony abnormalities are often present. The KTS syndrome is uncommon hence its approach has remained with poor understanding of its etiology and pathogenesis ${ }^{3}$. As our understanding of vascular anomalies and lesions have evolved, led to more recognition of the syndrome as a distinct entity. In the present case identification of syndrome led to a search for pulmonary embolism. The diagnosis often is done on the basis of history and examination result which is summarized as below.

1. Long standing varicose vein and vascular malformations

2. Soft tissue and bony hypertrophy involving one or more extremities

3. Vascular nevus

4. Syndactyly

5. Long standing lymphedema

6. High incidence of venous thromboembolism

7. Evidence of chronic thromboembolic pulmonary hypertension (CTEPH)

The pulmonary abnormalities associated with this syndrome include pulmonary lymphatic obstruction, pulmonary vein varicosities and cavernous hemangiomas of pleura leading to hemothorax. Vascular smooth muscle hyperplasia leading to lymphatic obstruction ${ }^{4}$. We did not notice evidence of DVT in lower extremities. The estimated incidence of pulmonary embolism in KTWS is reported to be from $14 \%$ to $22 \%{ }^{4}$. The cases of KTW syndrome who reveal arteriovenous communication indicate a bad prognosis. Hemangiomatosis is the most frequent finding in these patients. The vascular lesion may be unilateral or bilateral and can be present in 
the oral cavity, on the trunk, buttocks, limbs, head and / or neck. Cavernous hemangiomas, capillary hemangiomas, cutaneous hemangiomas, phlebectasia have been included in the vascular lesions of the syndrome. These findings are also present with Sturge weber syndrome causing diagnostic confusion. Coexistent cases with these two syndromes also have been reported ${ }^{5}$. The hypertrophy is yet another clinical finding that occurs at any age and may increase with age. The hypertrophy can be unilateral or bilateral. The osteohypertrophy may led to increase in length of extremity. Macrodactyly, polydactyly, syndactyly and oligodactyly can occur in this syndrome. The present case had bilateral limb hypertrophy with B/L polydactyly with elongated toes on either foot.

The etiology of this syndrome is still unknown but Koch suggested an autosomal dominant form of inheritance ${ }^{6}$. Others have excluded familial inheritance and proposed sporadic occurrence ${ }^{7}$. Etiological hypothesis is embryonic mesodermal changes which cause increase angiogenesis leading to increase vascularity flow and tissue hypertrophy. Another hypothesis is leathel mutation in a gene which explains why family links to disease exist.

The diagnosis of KTS is clinical, imaging for the venous system of the lower extremities is an important adjunctive study. The common findings include hypoplastic or absent deep venous system, venous obstruction by fibrous bands, and venous obstruction by adjacent arteries and incompetent valves. Contrast venography is ideal, magnetic resonance technique is an alternative technique. The present case had shown bilateral saphenofemoral

Incompetency and poor flow reversal on Valsalva in standing and lying down position either side on venous Doppler showing venous impediment flow. The management of KTS is based on specific type and severity of symptom and never a single pattern of treatment recommended. Surgical management in selected cases for varicose veins are carried out. Debulking operations are often discouraged as they often fail and worsen symptoms as lymphatic and venous channels are destroyed. Lack of normal venous drainage may be an etiologic factor in hypertrophied and elongated extremities.

Deep venous thrombosis is not uncommon in KTS, pulmonary embolisms are rare. There have been four previous English language case reports of pulmonary emboli in patients with KTS ${ }^{\mathbf{8}}$. The present patient had progressive dyspnea for long period similar to one described by Mikula et al in $1991{ }^{9}$. There are no firm treatment recommendations for treatment of thromboembolic phenomenon with KTS owing to lack of large experiences in the literatures. The logical approach remains with use of anticoagulation with or without caval filter placement.

\section{Conclusion}

Klippel Trenaunay weber syndrome is a rare disease of vascular and lymphatic system presenting with port wine nevus, vascular malformations, hypertrophy of both bony and soft tissues. The well-defined features of acute pulmonary hypertension or even CTEPH in particular developing in KTS cohort should be considered as alarming signal for occurrence of pulmonary thromboembolism. Based on the current finding we suggest that indication for screening for unexplained pulmonary hypertension development amongst patient with KTS who present with shortness of breath merits further investigations and should be screened for diagnosis of pulmonary embolism and aggressive treatment.

\section{REFERENCES}

[1]. James, William; Berger, Timothy; Elston, Dirk (2005). Andrews' Diseases of the Skin: Clinical Dermatology (10th ed). Saunders. p. 585. 0-7216-2921-0.

[2]. You, CK, Rees, J, Gillis, DA, et al (1983) Klippel-Trenaunay syndrome: a review. Can J Surg26,399-403.

[3]. Lindenauer SM. The Klippel-Trenaunay syndrome: varicosity, hypertrophy and haemangioma with no arteriovenous fistula. Ann Surg 1965; 162:303-314.

[4]. Adriane Gianlupi, Richart W. Harper,Denis M. Dwyre et al. Recurrent Pulmonary Embolism Associated With KlippelTrenaunay-Weber Syndrome. Chest.1999;115(4):1199-1201. doi:10.1378/chest.115.4.1199

[5]. Rose LF, Kaye D: Internal Medicine for Dentistry, St. Louis: The CV Mosby Co, 1991, pp 777-79.

[6]. Koch G: Eur klinik symptomatologie pathogeneses and erbpathologie des Klippel-TrenaunayoWeber-schen syndrom. Acta Genet Med Wochenschr 84:326-70, 1956.

[7]. Gorlin RJ, Pindborg JJ: Syndromes of the Head and Neck, 2nd Ed. New York: McGraw-Hill Book Co, 1976 , pp 412-17.

[8]. Awad AN, Yang DC, Girgis M, et al. Evaluation of 12. Klippel-Trenaunay syndrome with radionuclide total body angiography: a case report. Clin Nucl Med 1992;17: 866-70.

[9]. Mikula N Jr, Gupta SM, Miller M, Felder S. Klippel Trenaunay-Weber syndrome with recurrent pulmonary embolism. Clin Nucl Med 1991;16:253-5. 